\title{
Rapid characterization of the ultraviolet induced fiber Bragg grating complex coupling coefficient as a function of irradiance and exposure time
}

\author{
Gordon M. H. Flockhart, ${ }^{1, *}$ Geoffrey A. Cranch, ${ }^{1}$ and Clay K. Kirkendall ${ }^{2}$ \\ 1SFA, Inc., 2200 Defense Highway, Suite 405, Crofton, Maryland 21114, USA \\ 2U.S. Naval Research Laboratory, 4555 Overlook Avenue SW, Washington, D.C. 20375, USA \\ *Corresponding author: gordon.flockhart@nrl.navy.mil \\ Received 25 July 2007; accepted 30 September 2007; \\ posted 15 October 2007 (Doc. ID 85539); published 26 November 2007
}

\begin{abstract}
We report the application of optical frequency domain reflectometry and a discrete-layer-peeling inverse scattering algorithm to the spatial characterization of the UV induced complex coupling coefficient during fiber Bragg grating growth. The fiber grating is rapidly characterized using this technique to give irradiance dependent growth as a function of exposure time, thereby providing the complete characterization of the coupling coefficient in the form of a "growth surface," which is related to the fiber's photosensitivity. We compare measurements of fiber Bragg grating growth in SMF-28 when exposed to continuous wave $244 \mathrm{~nm}$ irradiation from 0 to $90 \mathrm{~W} \mathrm{~cm}^{-2}$ for exposure times up to $3230 \mathrm{~s}$ with a selection of other fibers including high germanium concentration fiber and erbium doped fiber. (C) 2007 Optical Society of America
\end{abstract}

OCIS codes: $\quad 060.0060,060.3738,060.2270,060.2410,120.3180,160.5335$.

\section{Introduction}

Fiber Bragg gratings (FBGs) are periodic variations of an optical fiber's core refractive index along the axis (length) of the optical fiber. This class of grating structures obeys a phase matching condition whereby energy is coupled from a forward propagating core mode to a backward propagating core mode. FBGs are highly versatile devices whose spectral amplitude, wavelength, and dispersion properties can be tailored by controlling numerous properties of the refractive index grating structure, including: fringe periodicity and chirp, fringe magnitude and apodization, and fringe tilt angle [1]. As a result of their versatility FBGs have found uses in many applications in optical fiber sensing [2] and as in-fiber devices for telecommunication applications [3]. FBG based devices such as multiband spectral filters [4] can require extremely complicated grating structures. The exacting specifications of these devices demand precise control of the refractive index grating

0003-6935/07/348237-07\$15.00/0

(C) 2007 Optical Society of America structure. FBGs can be fabricated by exposing the core of an optical fiber to a sinusoidal UV irradiance pattern along the length of the fiber formed by the interference between two UV laser beams [5,6]. Advanced fabrication techniques have been developed to allow the various parameters of the grating structure to be controlled during fabrication [7]. To design and fabricate sophisticated FBG devices grating modeling and synthesis algorithms are required, as well as precise knowledge of the optical fiber's refractive index change with applied optical radiation, commonly referred to as the fiber's photosensitivity.

To fabricate an FBG with a target spectral response it is necessary to determine the required complex coupling coefficient of the grating structure from the spectral response. This problem is commonly known as an inverse scattering problem, and one of the most promising techniques to calculate the grating structure from the target spectrum is the discrete-layer-peeling inverse scattering algorithm [8-10]. This algorithm can be combined with a specific set of conditions to ensure the FBG's target spectrum can be obtained by a physical grating structure [11]. The final step of the process is to write a refrac- 
tive index modulation in the optical fiber to give the desired complex coupling coefficient profile, ideally in a one-step process. Therefore the fiber's photosensitivity must be known and is typically described as the refractive index change for a given exposure irradiance. In the simplest case the refractive index modulation shape is assumed to be sinusoidal, from which the coupling coefficient can be directly calculated. If we approximate the refractive index modulation $n$ along the longitudinal axis of the fiber $x$ to be sinusoidal, the perturbation can be described by [11]

$$
n-n_{e f f}=\Delta n_{a c}(x) \cos \left(\frac{2 \pi}{\Lambda} z+\theta(x)\right)+\Delta n_{d c}(x)
$$

where $n_{\text {eff }}$ is the effective index of the guided mode of the nascent fiber; $\Delta n_{a c}(x)$ and $\Delta n_{d c}(x)$ are the slowly varying " $a c$ " and " $d c$ " components of the refractive index modulation; $\Lambda$ is the grating's spatial period; and $\theta(x)$ is the grating spatial phase. The coupling coefficient magnitude is directly related to the ac component of the refractive index variation, $\Delta n_{a c}(x)$, by [12]

$$
|q(x)|=\frac{\pi \eta}{\lambda_{B}} \Delta n_{a c}(x),
$$

where $\lambda_{B}=2 n_{\text {eff }} \Lambda$ is the design Bragg wavelength and $\eta$ is the guided mode overlap factor. The phase of the coupling coefficient describes the grating's phase envelope. This is a combination of the geometrical phase $\theta(x)$ and the phase due to the $d c$ refractive index change $\Delta n_{d c}(x)$ and is described by

$$
\arg [q(x)]=\theta(x)-\frac{4 \pi \eta}{\lambda_{B}} \int^{x} \Delta n_{d c}\left(x^{\prime}\right) \mathrm{d} x^{\prime}+\frac{\pi}{2}
$$

0

Although the refractive index perturbation represents the physical change in the optical fiber when the grating is fabricated, it is the complex coupling coefficient of the grating structure that describes the magnitude and phase of the mode coupling. In materials with nonlinear photosensitivity, i.e., the refractive index change is a nonlinear function of the incident irradiance, the refractive index modulation shape is no longer sinusoidal and a modified coupling coefficient must therefore be determined $[13,14]$.

Miller et al. proposed a three-dimensional growth surface describing the fiber's photosensitivity as a function of irradiance and exposure time, thereby being able to predict the refractive index modulation shape and hence the modified coupling coefficient [14]. Recently, characterization of the growth surface of a germanosilicate fiber was reported using an interferometric approach to directly measure the refractive index change when exposed to fringeless UV radiation [15]. However characterization of the fiber's photosensitivity in terms of the UV induced complex coupling coefficient obviates the need to know the precise refractive index modulation shape and hence the need to calculate a modified coupling coefficient. Knowledge of the spatially varying complex coupling coefficient, design wavelength, and $n_{\text {eff }}$ are sufficient to completely characterize the spectral properties of the FBG over the first-order resonance regime.

In this paper we propose a technique to rapidly characterize a fiber's photosensitivity in terms of the UV induced coupling coefficient as a function of incident UV irradiation and exposure time. Characterization in terms of the UV induced coupling coefficient, rather than the UV induced refractive index change, is advantageous for FBG design and fabrication as it provides a direct measure of the coupling strength of the grating structure realized in the fiber. This measurement therefore includes effects such as the contribution of higher diffraction orders for direct phase mask inscription, which can degrade the UV fringe pattern or any reduction in UV fringe visibility in interferometric writing setups. We report the full characterization of the coupling coefficient growth surface of SMF-28 for exposure to $244 \mathrm{~nm}$ radiation from 0 to $\sim 90 \mathrm{~W} \mathrm{~cm}^{-2}$ and a total accumulated exposure time of $3230 \mathrm{~s}$. After characterization of the beam profile and alignment of the optics, a typical measurement will take $\sim 2-3 \mathrm{~h}$ depending on the required total fluence. The measurement is fully automated and requires no user intervention. We also report measurements of a high germanium-doped fiber and three erbium-doped fibers for comparison.

\section{Experimental Setup}

To characterize the optical fiber's growth surface as a function of irradiance and exposure time, we perform a stepwise growth of a FBG exposed to an incident UV beam with a nonuniform irradiance profile. Spatial characterization of the complex coupling coefficient and knowledge of the UV beam's irradiance profile allows the irradiance dependent growth to be determined. As a result the fiber grating's growth can be rapidly characterized as a function of irradiance and exposure time in a single experiment. To demonstrate this technique a number of FBG exposures were characterized in five different optical fibers.

A diagram of the optical setup is shown in Fig. 1. A continuous wave frequency-doubled argon-ion laser

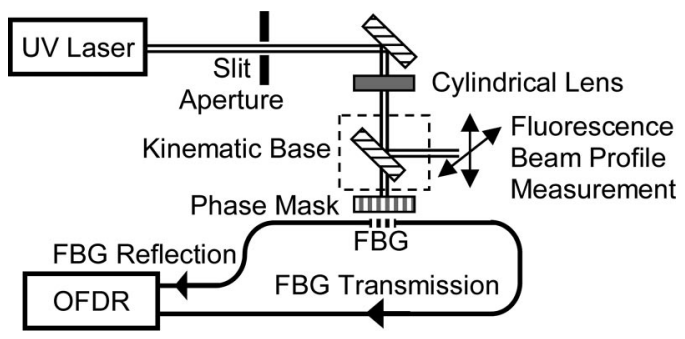

Fig. 1. Schematic of experimental setup used to characterize UV beam, expose optical fiber, and characterize FBG's complex reflectivity and transmission spectra. 
( $\lambda=244 \mathrm{~nm}$ and output power $P_{\text {out }}=125 \mathrm{~mW}$ ) is used to expose the optical fiber through a uniform period phase mask $\left(\Lambda_{\mathrm{PM}}=1067 \mathrm{~nm}\right)$. The laser beam is expanded ( $6 \times$ telescope not shown) and focused using a cylindrical lens to a line with a $1 / e^{2}$ beam width of $\sim 14 \mu \mathrm{m}$ (full width $28 \mu \mathrm{m}$ ), and a slit aperture truncates the sidelobes of the beam leaving an approximately Gaussian profile with a $1 / e^{2}$ beam width of $2.4 \mathrm{~mm}$. The FBGs are written using a static full field exposure, and the focused beam shape is characterized before each series of exposures. The focused beam profile is measured by scanning an optical fiber through the beam waist. The emitted $410 \mathrm{~nm}$ fluorescence [16] guided by the fiber is detected using a silicon photodiode and is recorded as a function of position. A horizontal and a vertical beam profile are recorded. The fluorescence intensity decays as a function of UV exposure, therefore the scan speed must be set appropriately to avoid measurement errors in the beam profile. The total power incident on the phase mask is measured $(P=70 \mathrm{~mW})$ and used to calculate the beam irradiance. We assume the measured horizontal and vertical beam profiles represent the beam shape across the full beam width, thus we approximate the full beam by matrix multiplication of the two beam profiles. The beam irradiance is calculated by numerical integration of the beam shape and scaled by the \pm 1 diffraction efficiencies of the phase mask $(\sim 38 \%)$ and the Fresnel reflection. Typical horizontal and vertical beam irradiance profiles are shown in Fig. 2 .

The fiber is exposed for a set period of time increasing from $50 \mathrm{~ms}$ up to $600 \mathrm{~s}$, after which the grating is allowed to decay for $60 \mathrm{~s}$ [17]. The complex reflectivity of the FBG is then measured using an optical frequency domain reflectometer (OFDR) [18]. Preliminary measurements were performed using an in-house OFDR [19], however the fringe amplitude of the OFDR depended on the polarization state incident on the FBG under test. A polarization controller was used to

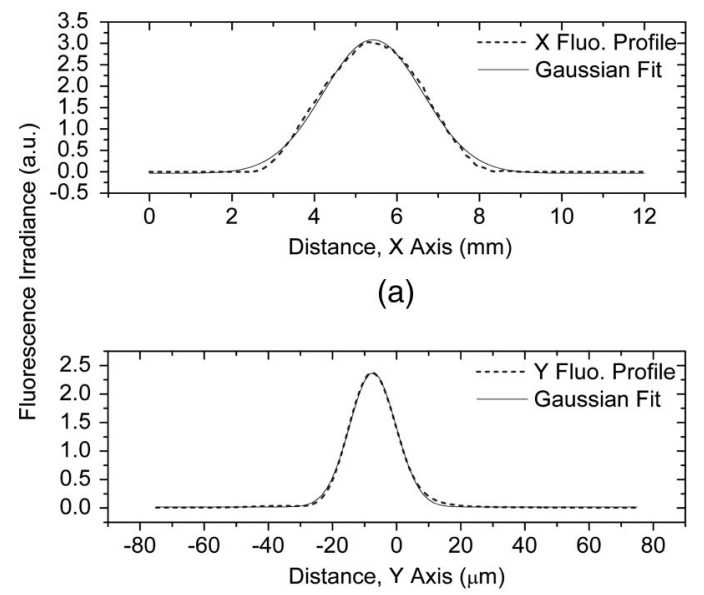

(b)

Fig. 2. Measured fluorescence profiles of UV beam waist with Gaussian fits. (a) Horizontal $1 / e^{2}$ beam width of $2.4 \mathrm{~mm}$ $\left(R^{2}=0.996\right)$. (b) Vertical $1 / e^{2}$ beam width of $14 \mu \mathrm{m}\left(R^{2}=0.999\right)$. maximize the fringe visibility at the start of an experiment, and the incident polarization state was assumed not to change during the course of the experiment. Any drift in the incident polarization state would affect the fringe visibility and hence the measured reflectivity accuracy. Also in the case of a birefringent FBG a change in the incident polarization state would result in a change of the measured FBG spectrum. Therefore to eliminate these errors a commercial OFDR (Luna OVA CTe) capable of polarization resolved measurements has been used for the results presented in this paper [20]. The FBG's transmission spectrum is also measured using the OFDR to accurately scale the measured reflectivity thereby taking into account any connector or fusion splice losses. The FBG's complex coupling coefficient is calculated from the polarization averaged complex reflectivity using the discrete layer peeling algorithm. Recently a polarization-resolved discrete layer peeling algorithm has been developed [21] and used to investigate UV induced birefringence. Potentially this could be applied to measurements reported in this paper.

The measured UV beam irradiance profile along the axis of the fiber is resampled to the same spatial resolution $(38.4 \mu \mathrm{m})$ of the reconstructed coupling coefficient. The magnitude of the coupling coefficient is aligned to the irradiance profile data by a correlation to allow the growth at a specific spatial location to be related to the incident UV irradiance. However it was necessary to perform this correlation for each characterization scan due to variations in the FBG's measured position as a result of environmental perturbations in the downlead to the FBG under test. The FBG forms a reflector in one arm of an interferometer, therefore environmental perturbations in the downlead can affect the phase difference between the arms, and hence the measured position of the FBG's impulse response from which the complex reflectivity is calculated by Fourier transformation. The fiber downleads to the OFDR ( $\sim 5 \mathrm{~m}$ in length) were insulated from environmental perturbations wherever possible. However the repeatability of the OFDR was measured to range from $\pm 25 \mu \mathrm{m}$ for measurements in rapid succession $(\sim 30 \mathrm{~s}$ intervals $)$ to $\pm 100 \mu \mathrm{m}$ over the course of an experiment $(\sim 1.75 \mathrm{~h})$. Also the UV laser beam pointing stability affects the FBG growth at a particular position. Any beam movement along the axis of the optical fiber will expose a specific position in the fiber to different irradiances. Any beam movement perpendicular to the fiber's axis will lead to a misalignment affecting all positions in the fiber reducing the incident irradiance. These effects can be minimized by allowing the laser to warm up sufficiently to ensure stable operation.

\section{Results}

The photosensitivity of SMF-28 in its nascent form was characterized. The magnitude of the complex coupling coefficient, $|q|$, versus position for SMF-28 after centering by correlation with the beam profile is shown in Fig. 3 for increasing exposure times. The 


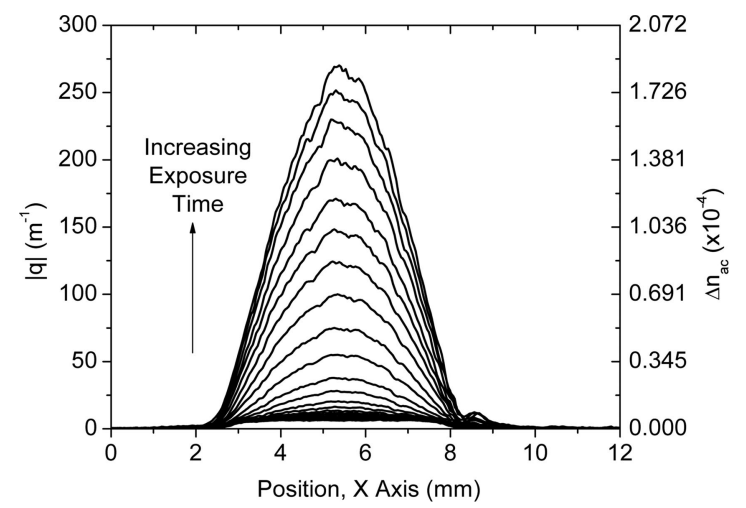

Fig. 3. Measured coupling coefficient magnitude after alignment with fluorescence profile and $\Delta n_{a c}(x)$ for an equivalent sinusoidal refractive index modulation.

magnitude of the coupling coefficient is directly proportional to $\Delta n_{a c}(x)$ for an equivalent sinusoidal refractive index modulation as described in Eq. (2). The design Bragg wavelength $\lambda_{B}$ was calculated, using the centroid wavelength of the reflectivity spectrum for the first exposure, to be $1543.830 \mathrm{~nm}$. The guided mode overlap factor $\eta$ was calculated using the Gaussian mode approximation by

$$
\eta=1-\exp \left(\frac{-2 a^{2}}{\omega^{2}}\right)
$$

where $a$ is the core radius and $\omega$ is the mode radius. Using values for SMF-28 [22], the core radius is $4.1 \mu \mathrm{m}$ and mode field radius is $5.2 \mu \mathrm{m}$, gives $\eta$ to be 0.71 . The $a c$ index modulation $\Delta n_{a c}(x)$ was calculated using these values from the magnitude of the coupling coefficient and is added as a second dependent variable axis in Figs. 3 and 4.

Since we also have measured the irradiance profile of the UV beam, these data can be combined to investigate the induced coupling coefficient magnitude for different UV irradiances and as a function of exposure time. The growth of the FBG can be plotted as a function of irradiance and is shown in Fig. 4 for a

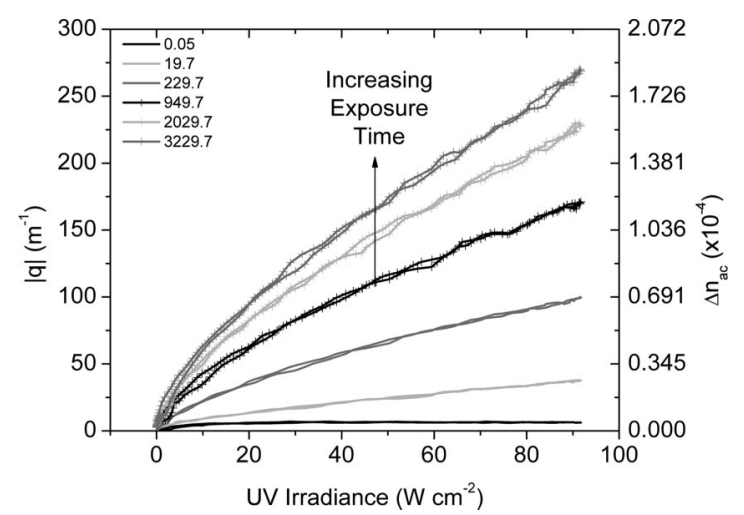

Fig. 4. Measured coupling coefficient magnitude versus irradiance for select exposure times, and $\Delta n_{a c}(x)$ for an equivalent sinusoidal refractive index modulation.

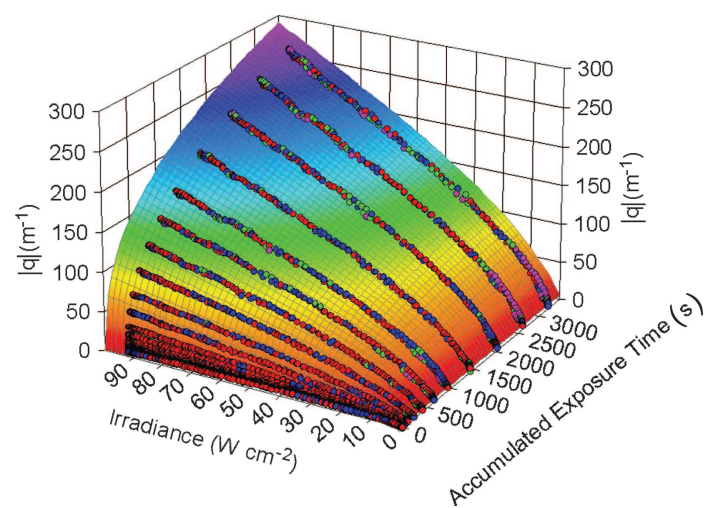

Fig. 5. (Color online) Measured $|q|$ as a function of accumulated exposure time and incident UV irradiance (circles) and 3D surface fit of Eq. (5). (The color fill of the data points describes the magnitude of the residual compared to the standard error of the fit: red $<1$ standard error, blue $<2$ standard error, green $<3$ standard error, and magenta $>3$ standard error.

selected number of exposure times. For the initial exposure of $0.05 \mathrm{~s}$ the measured coupling coefficient magnitude is approximately constant for irradiances above $30 \mathrm{~W} \mathrm{~cm}^{-2}$. As the exposure time increases, the UV induced coupling coefficient is observed to increase as a function of irradiance. To visualize the growth of $|q|$, all of the acquired data can be plotted in three dimensions as a growth surface. Figure 5 shows the measured data points, circles, for nonhydrogen loaded SMF-28 characterizing the UV induced coupling coefficient magnitude as a function of incident irradiance (from 0 to $92 \mathrm{~W} \mathrm{~cm}^{-2}$ ) and accumulated exposure time (from 0.050 to $3229.7 \mathrm{~s}$ ). This surface completely describes the fiber's photosensitivity in terms of the magnitude of the complex coupling coefficient and therefore can be directly used with grating synthesis algorithms to determine the required exposure conditions to fabricate an FBG with a desired spectral response. To use this surface for grating design it is desirable to describe the surface analytically. Therefore we must perform a surface fit to the measured data. Previous photosensitivity measurements have indicated a power law growth [23]. However it has also been suggested the growth exhibits a sigmoidal behavior and exhibits power law characteristics only over a small region of the growth curve [24,25]. It is beyond the scope of this paper to investigate the photosensitivity mechanisms contributing to the measured growth curve. The fundamental understanding of the UV induced refractive index change is still under investigation $[26,27]$ and references therein. In this work we report the rapid characterization of the UV induced growth and determine an analytical description of it for FBG fabrication purposes. Therefore to investigate the growth function, $|q|$ is plotted for different irradiance values versus the accumulated exposure time on a log-log plot, see Fig. 6. The growth does not follow a power law for all exposure times but does exhibit power law behavior for longer exposure times and at higher irradiances. 


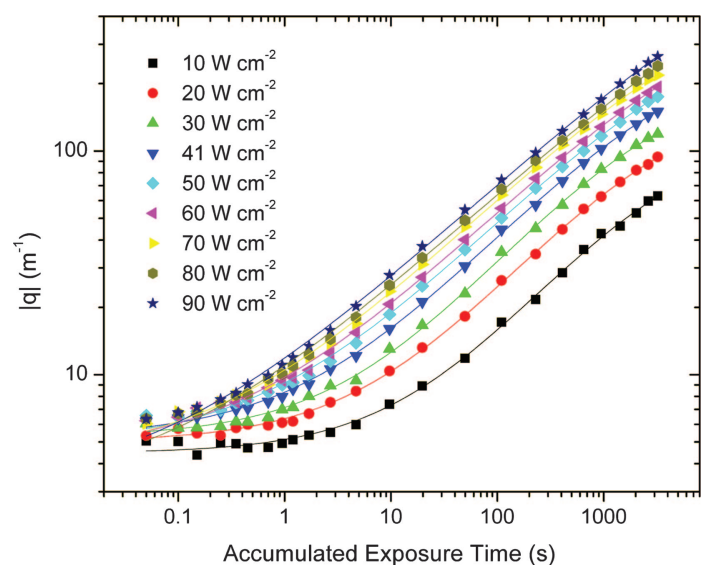

Fig. 6. (Color online) Log-log plot of $|q|$ versus time for select irradiance values (data points), with individual logistic fits (curve).

A logistic function was found to best describe grating growth for specific irradiance values versus time, and these fits are shown in Fig. 6. These fits agree very well for the individual data sets, therefore a surface fit based on this logistic function was investigated to describe the growth as a function of irradiance and time. A logistic function of the form

$$
|q(I, t)|=\frac{A_{1}(I)-A_{2}(I)}{1+[t / \tau(I)]^{\alpha(I)}}+A_{2}(I)
$$

where

$$
\begin{aligned}
A_{1}(I) & =a_{11} I^{2}+a_{12} I+a_{13}, \\
A_{2}(I) & =a_{21} I^{2}+a_{22} I+a_{23}, \\
\tau(I) & =\tau_{1} I+\tau_{2} I+\tau_{3} \\
\alpha(I) & =\alpha_{1} I+\alpha_{2}
\end{aligned}
$$

was determined to be the best description of the growth surface. The coefficient of determination, $R^{2}$, for the surface fit was 0.9994 , and the coefficients of the logistic surface fit are given in Table 1 with their

Table 1. Coefficients for Logisitic Eq. (5)

\begin{tabular}{ccc}
\hline & Value & $\begin{array}{c}\text { Standard } \\
\text { Error }\end{array}$ \\
\hline$a_{11}$ & $-1.45 \times 10^{-3}$ & $5.10 \times 10^{-5}$ \\
$a_{12}$ & 0.138 & $4.39 \times 10^{-3}$ \\
$a_{13}$ & 1.17 & $5.80 \times 10^{-2}$ \\
$a_{21}$ & $-1.9 \times 10^{-3}$ & $2.92 \times 10^{-3}$ \\
$a_{22}$ & 12.6 & 0.232 \\
$a_{23}$ & 1.1 & 0.251 \\
$\tau_{1}$ & 8.4 & 0.401 \\
$\tau_{2}$ & 431 & 30.9 \\
$\tau_{3}$ & 1570 & 125 \\
$\alpha_{1}$ & $-7.9 \times 10^{-4}$ & $3.19 \times 10^{-5}$ \\
$\alpha_{2}$ & 0.5123 & $3.08 \times 10^{-3}$ \\
\hline
\end{tabular}

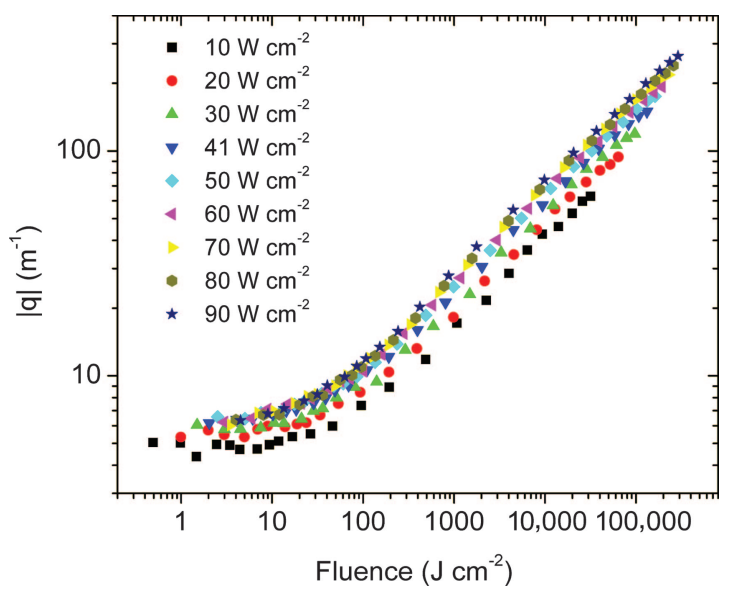

Fig. 7. (Color online) Growth versus cumulative fluence for select UV irradiances.

respective standard errors. The surface fit is shown in Fig. 5.

Although it is clear that the change in the UV induced coupling coefficient is irradiance dependent, we can also investigate the growth as a function of the cumulative fluence. The cumulative fluence for all irradiance values is calculated and is plotted for irradiance values between 10 and $90 \mathrm{~W} \mathrm{~cm}{ }^{-2}$ in Fig. 7 . This plot clearly shows that the growth is not purely a function of cumulative fluence, but is dependent on the incident UV irradiance. The resultant UV induced coupling coefficient for exposure conditions with equal fluences depends on the irradiance level of the illuminating radiation.

The study of the fiber's photosensitivity has concentrated on the magnitude of the coupling coefficient, which is proportional to $\Delta n_{a c}$ for an equivalent sinusoidal grating. However the phase of the coupling coefficient can also be used. The phase described in Eq. (3) describes any excess phase in the grating structure due to a physical chirp of the grating period $\theta(x)$ or due to a change of $\Delta n_{d c}$. These two terms have the same effect on the grating's response, and therefore cannot be distinguished between unless a priori knowledge of the grating's structure is used. In this work a uniform period phase mask is used to expose the fiber, therefore we make the assumption that there is no physical chirp of the grating period, i.e., $\theta(x)=0$. Any excess phase will be the result of a change in $\Delta n_{d c}(x)$ which can be calculated by differentiation of Eq. (3) with respect to $x$, yielding

$$
\Delta n_{d c}(x)=-\frac{\lambda_{B}}{4 \pi \eta} \frac{\mathrm{d} \arg [q(x)]}{\mathrm{d} x},
$$

The phase of the complex coupling coefficient is differentiated and smoothed using a Savitzky-Golay filter to remove excess noise and is plotted in Fig. 8. It is clear that the phase information exhibits increased noise compared with the amplitude information, especially where the grating structure is very weak, i.e., at the edges. In the center of the grating 


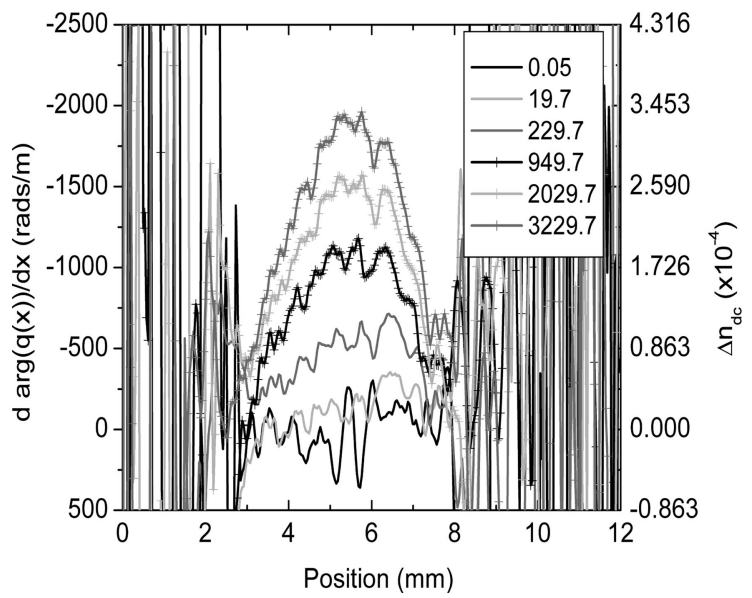

Fig. 8. Differentiated and smoothed phase of complex coupling coefficient for select exposure times.

the differentiated phase indicates a change in $\Delta n_{d c}$ as a function of position and of similar shape to the UV beam irradiance. This is expected for illumination with a Gaussian beam and gives rise to self-chirping of the grating structure. It is also interesting to compare the magnitudes of the $a c$ and $d c$ refractive index changes. The peak $\Delta n_{a c}$ and $\Delta n_{d c}$ for the accumulated exposure time of $3229.7 \mathrm{~s}$ are $1.866 \times 10^{-4}$ and $3.386 \times 10^{-4}$, respectively. The visibility of the fringes can be calculated by the ratio of $\Delta n_{a c}: \Delta n_{d c}$ and is 0.55 . The reduction from unity fringe visibility may be attributed to the contribution of the zeroth-order beam, and higher-order diffraction beams, reducing the interference fringe's visibility as well as modifying the fringe modulation shape [28]. Also the nonlinear photosensitivity affects the refractive index modulation shape, which in effect changes the fringe visibility in comparison to a sinusoidal modulation shape described by $\Delta n_{a c}$.

Finally, to compare the response of a number of fibers we have plotted in Fig. 9, the peak $|q|$ versus accumulated exposure time for the following fibers:

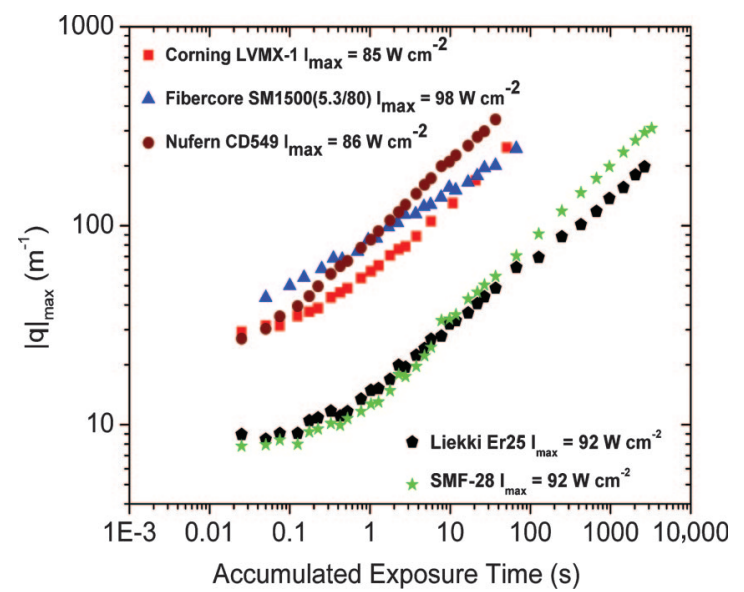

Fig. 9. (Color online) Comparison of the peak UV induced coupling coefficient in a selection of optical fibers and the corresponding exposure conditions.
Liekki Er25, Fibercore SM1550 (5.3/80), Nufern CD549 (also called DD17), and Corning LVMX-1 (no longer produced). The Fibercore SM1500 fiber is a high Ge-concentration fiber and exhibits considerably higher photosensitivity compared to SMF-28. The remaining fibers are erbium doped fibers and the increased photosensitivity of these fibers is of particular interest for the fabrication of FBG defined fiber lasers. Nufern's erbium doped fiber, CD549, exhibits the highest UV induced coupling coefficient of the erbium fibers tested.

\section{Conclusions}

We have demonstrated rapid characterization of FBG growth by the use of OFDR and discrete layer peeling to measure the complex coupling coefficient along the length of the grating during stepwise exposure to a UV beam with a Gaussian profile. These measurements are combined with a measurement of the UV writing beam irradiance profile to determine the growth as a function of irradiance and exposure time, thereby providing an experimental determination of the growth surface for the fiber under test. The measured growth surface for SMF-28 is described in terms of the complex coupling coefficient magnitude. The complex coupling coefficient is related to the $a c$ and $d c$ refractive index changes in the fiber according to Eqs. (2) and (3), however it is not a true measurement of the fiber's photosensitivity in terms of the UV induced refractive index change. Characterization in terms of the coupling coefficient is beneficial for grating design and prevents the need to determine the associated coupling coefficient for nonsinusoidal refractive index modulation shapes. Also in comparison to photosensitivity measurements using fringeless UV illumination, direct determination of the coupling coefficient realized in the fiber also includes the efficiency of the writing setup.

The surface clearly shows the nonlinear irradiance and time dependence of the growth and was found to be best described analytically by a logistic function. A surface fit was performed to the data, and the coefficient of determination, $R^{2}$, was 0.9994 . This technique was applied to the characterization of a selection of fibers and their growth characteristics were compared.

The authors thank Gary A. Miller for provision of the SM1500 fiber to be tested and for the useful discussions regarding the growth surface characterization.

\section{References}

1. T. Erdogan, "Fiber grating spectra," J. Lightwave Technol. 15, 1277-1294 (1997).

2. A. D. Kersey, M. A. Davis, H. J. Patrick, M. LeBlanc, K. P. Koo, C. G. Askins, M. A. Putnam, and E. J. Friebele, "Fiber grating sensors," J. Lightwave Technol. 15, 1442-1463 (1997).

3. C. R. Giles, "Lightwave applications of fiber Bragg gratings," J. Lightwave Technol. 15, 1391-1404 (1997).

4. J. Bland-Hawthorn, M. Englund, and G. Edvell, "New approach to atmospheric $\mathrm{OH}$ suppression using an aperiodic fibre Bragg grating," Opt. Express 12, 5902-5909 (2004). 
5. G. Meltz, W. W. Morey, and W. H. Glenn, "Formation of Bragg gratings in optical fibers by a transverse holographic method," Opt. Lett. 14, 823-825 (1989).

6. K. O. Hill, B. Malo, F. Bilodeau, D. C. Johnson, and J. Albert, "Bragg gratings fabricated in monomode photosensitive optical fiber by UV exposure through a phase mask," Appl. Phys. Lett. 62, 1035-1037 (1993).

7. A. Asseh, H. Storoy, B. E. Sahlgren, S. Sandgren, and R. A. H. Stubbe, "A writing technique for long fiber Bragg gratings with complex reflectivity profiles," J. Lightwave Technol. 15, 14191423 (1997).

8. R. Feced, M. N. Zervas, and M. A. Muriel, "An efficient inverse scattering algorithm for the design of nonuniform fiber Bragg gratings," IEEE J. Quantum Electron. 35, 1105-1115 (1999).

9. L. Poladian, "Simple grating synthesis algorithm," Opt. Lett. 25, 787-789 (2000).

10. J. Skaar, L. G. Wang, and T. Erdogan, "On the synthesis of fiber Bragg gratings by layer peeling," IEEE J. Quantum Electron. 37, 165-173 (2001).

11. J. Skaar and O. H. Waagaard, "Design and characterization of finite-length fiber gratings," IEEE J. Quantum Electron. 39, 1238-1245 (2003).

12. J. Skaar, "Synthesis and characterization of fiber Bragg gratings," Ph.D. dissertation (The Norwegian University of Science and Technology, 2000).

13. J. A. Besley, L. Reekie, C. Weeks, T. Wang, and C. Murphy, "Grating writing model for materials with nonlinear photosensitive response," J. Lightwave Technol. 21, 2421-2428 (2003).

14. G. A. Miller, C. G. Askins, and E. J. Friebele, "Modified F-matrix calculation of Bragg grating spectra and its use with a novel nonlinear index growth law," J. Lightwave Technol. 24, 2416-2427 (2006).

15. G. A. Miller, C. G. Askins, G. A. Cranch, and E. J. Friebele, "Early index growth in germanosilicate fiber upon exposure to continuous wave ultraviolet light," J. Lightwave Technol. 25, 1034-1044 (2007).

16. D. L. Williams, S. T. Davey, R. Kashyap, J. R. Armitage, and B. J. Ainslie, "Direct observation of UV induced bleaching of 240-nm absorption-band in photosensitive germanosilicate glass-fibers," Electron. Lett. 28, 369-371 (1992).

17. T. Erdogan, V. Mizrahi, P. J. Lemaire, and D. Monroe, "Decay of ultraviolet-induced fiber Bragg gratings," J. Appl. Phys. 76, 73-80 (1994).

18. Luna Technologies, "Optical Vector Analyzer CTe," http:// www.lunatechnologies.com/products/ova/files/DATASHEET_ OVACTe.pdf.

19. G. M. H. Flockhart, G. A. Cranch, and C. K. Kirkendall, "Characterization of fiber Bragg grating growth using optical frequency domain reflectometry and layer-peeling," in Bragg Gratings, Poling \& Photosensitivity/30th Australian Conference on Optical Fibre Technology (BGPP/ACOFT) (2005), pp. 76-78.

20. M. Froggatt, "Distributed measurement of the complex modulation of a photoinduced Bragg grating in an optical fiber," Appl. Opt. 35, 5162-5164 (1996).

21. O. H. Waagaard, "Polarization-resolved spatial characterization of birefringent fiber Bragg gratings," Opt. Express 14, 4221-4236 (2006).

22. Corning, "SMF-28e optical fiber product information," http:// www.corning.com.

23. H. Patrick and S. L. Gilbert, "Growth of Bragg gratings produced by continuous-wave ultraviolet-light in optical-fiber," Opt. Lett. 18, 1484-1486 (1993).

24. B. Poumellec, "Links between writing and erasure (or stability) of Bragg gratings in disordered media," J. Non-Cryst. Solids 239, 108-115 (1998).

25. J. Canning, "The characteristic curve and site-selective laser excitation of local relaxation in glass," J. Chem. Phys. 120, 9715-9719 (2004).

26. M. Douay, W. X. Xie, B. LeConte, T. Taunay, P. Bernage, P. Niay, P. Cordier, J. F. Bayon, H. Poignant, and E. Delevaque, "Progress in silica optical fibre photosensitivity," Ann. Telecommun. 52, 543-556 (1997).

27. M. Kristensen, "Ultraviolet-light-induced processes in germaniumdoped silica," Phys. Rev. B 6414, 144201 (2001).

28. Z. S. Hegedus, "Contact printing of Bragg gratings in optical fibers: rigorous diffraction analysis," Appl. Opt. 36, 247-252 (1997). 